\title{
WestVirginiaUniversity
}

THE RESEARCH REPOSITORY @ WVU

Graduate Theses, Dissertations, and Problem Reports

2015

\section{The Influence of Memory Enhancement Techniques on Children's Testimony}

Ria M. Travers

Follow this and additional works at: https://researchrepository.wvu.edu/etd

\section{Recommended Citation}

Travers, Ria M., "The Influence of Memory Enhancement Techniques on Children's Testimony" (2015). Graduate Theses, Dissertations, and Problem Reports. 6828.

https://researchrepository.wvu.edu/etd/6828

This Dissertation is protected by copyright and/or related rights. It has been brought to you by the The Research Repository @ WVU with permission from the rights-holder(s). You are free to use this Dissertation in any way that is permitted by the copyright and related rights legislation that applies to your use. For other uses you must obtain permission from the rights-holder(s) directly, unless additional rights are indicated by a Creative Commons license in the record and/ or on the work itself. This Dissertation has been accepted for inclusion in WVU Graduate Theses, Dissertations, and Problem Reports collection by an authorized administrator of The Research Repository @ WVU.

For more information, please contact researchrepository@mail.wvu.edu. 


\title{
The Influence of Memory Enhancement Techniques on
}

Children's Testimony

\author{
Ria M. Travers, M.S. \\ Dissertation submitted \\ to the Eberly College of Arts and Sciences \\ at West Virginia University \\ in partial fulfillment of the requirements for the degree of \\ Doctorate of Philosophy in \\ Clinical Psychology
}

Elisa Krackow, Ph.D., Chair

Steven Kinsey, Ph.D.

Cheryl McNeil, Ph.D.

Christine Rittenour, Ph.D.

Constance Toffle, Ph.D.

Department of Psychology

Morgantown, West Virginia

2015

Keywords: source monitoring training, cognitive interview, children's eyewitness testimony Copyright 2015 Ria Travers 


\section{ABSTRACT \\ The Influence of Memory Enhancement Techniques on Children's}

\section{Testimony}

\section{Ria Travers, M.S.}

Eyewitness testimony is often key evidence in court cases, but it is often difficult to obtain accurate eyewitness memories (Loftus, Miller, \& Burns, 1978). This is especially true when this testimony must be acquired from preschool children because young children have been found to be susceptible to suggestive interviewing techniques (Krackow \& Lynn, 2003). Several aspects of children's cognitive capabilities have been found to influence their suggestibility, including their source monitoring abilities, or their abilities to trace the source of memories (Ceci $\&$ Bruck, 1993). Therefore, source monitoring ability provides a possible point of intervention to decrease children's suggestibility. Source monitoring training protocols have been developed, tested, and shown to decrease children's suggestibility (Giles, Gopnik, \& Heyman, 2002). Another intervention that has been developed and proven to improve children's eyewitness memory is the revised cognitive interview (Fisher \& Geiselman, 1992). This method utilizes a number of memory enhancement techniques in a structured interview protocol to obtain children's eyewitness memory. Although both source monitoring training and the revised cognitive interview have been shown to improve recall, they have never been compared directly. The current study utilized both memory enhancement techniques and compare them to a nointervention control condition to assess the influence of these techniques on children's recall and suggestibility. Thirty-seven 4- and 5-year-old children were randomized to the three conditions. There was no effect of source monitoring training or the cognitive interview on correct answers to leading questions or free recall memory reports. The results indicate that source monitoring training was not effective in decreasing suggestibility and the cognitive interview was not effective in obtaining greater free recall in the current study. Implications for forensic interviewing are discussed. 


\section{Table of Contents}

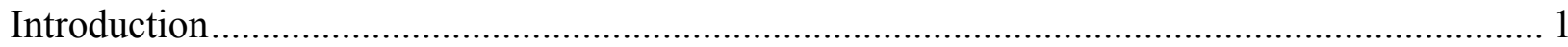

Reasons for Age Differences in Suggestibility ................................................................ 2

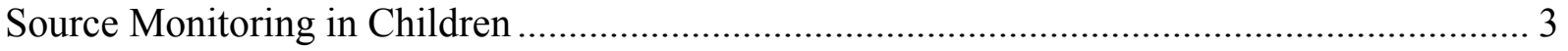

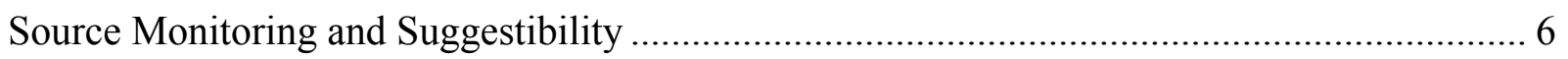

Suggestibility Reduction via Source Monitoring Training ............................................ 7

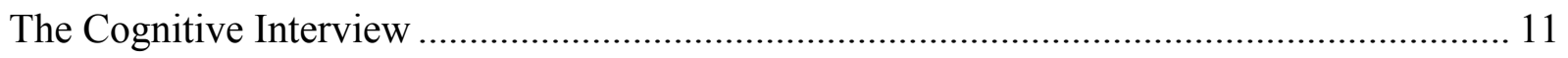

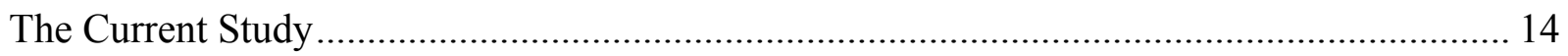

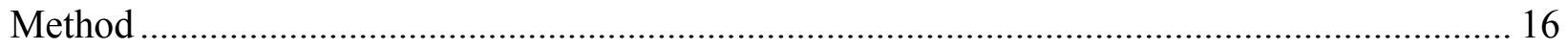

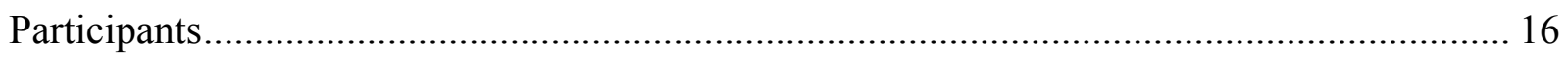

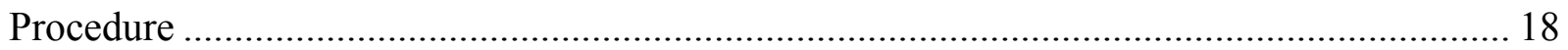

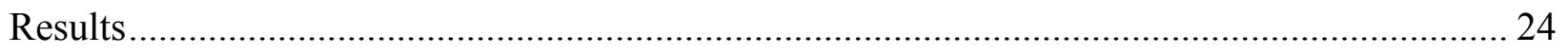

"Yes/no" Questions Accuracy: Misleading and Correctly Leading Questions ....................... 25

Free Recall Memory Accuracy: Correct and Incorrect Recall........................................... 26

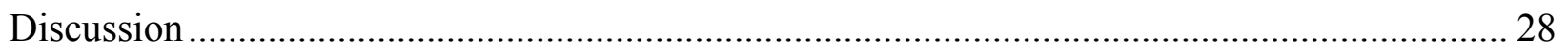

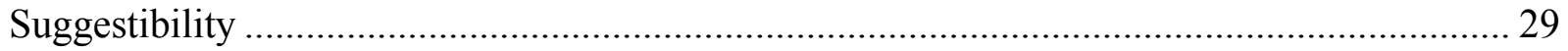

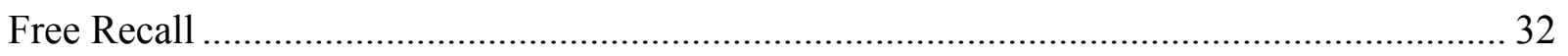

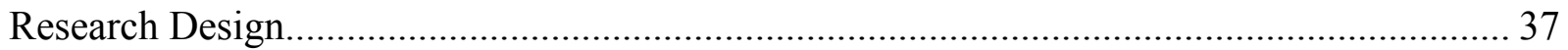

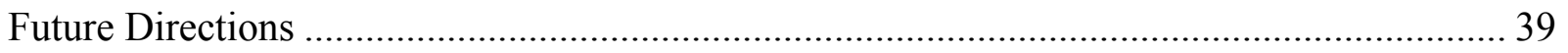

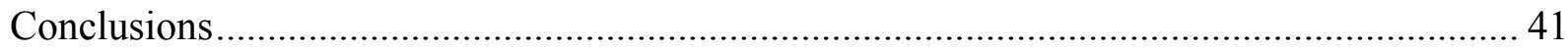

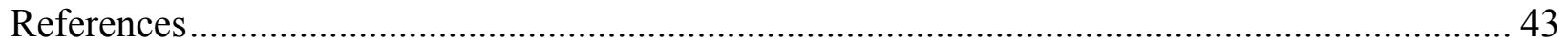




\section{Introduction}

Eyewitness testimony can be the most significant and compelling evidence to be submitted in a court case, and therefore it may greatly impact the eventual verdict and the future outcomes for individuals involved with the case (Geiselman, Fisher, MacKinnon, \& Holland, 1985). Consequently, it is important that eyewitness testimony is accurate and memory reports are complete (Geiselman, Fisher, MacKinnon, \& Holland, 1986). Obtaining accurate eyewitness testimony may be difficult, especially with young children who compared to older children and adults provide the least amount of information in response to open-ended questions (Krackow \& Lynn, 2010). Young children also have increased susceptibility to incorporating details from suggestive interviewing techniques into their memory reports compared to adults (Ceci \& Bruck, 1993).

Numerous studies in the literature point to the suggestibility of young children when suggestive interviewing techniques have been used. For example, Krackow and Lynn (2003) found that preschool children responded at chance rates to leading specific questions about bodily touch (e.g., "Amy touched your bottom, didn't she?" pg. 592). On the other hand, young children can be quite accurate in their memory reports when interviewed using less suggestive techniques (Krackow \& Lynn, 2003). Because research shows that investigative interviewers frequently interview using suggestive techniques even when trained not to do so (Warren, Woodall, Thomas, Nunno, Keeney, Larson, \& Stadfeld, 1999), it is imperative that researchers focus efforts on methods to reduce suggestibility in young children (Krackow \& Lynn, 2010). The current study compared the efficacy of two methods used to reduce suggestibility and improve recall in preschool children. One method will focus on source monitoring ability, one of the main explanations for young children's suggestibility. The second method will focus on 
the cognitive interview. Both of these will be compared to a control condition. However, before describing these two methods in more detail, reasons for age differences in suggestibility will be discussed.

\section{Reasons for Age Differences in Suggestibility}

Cognitive factors can greatly impact children's suggestibility (Ceci \& Bruck, 1993). Children's cognitive abilities develop over time, and therefore the developmental status of their cognitive skills may influence their suggestibility (Ceci \& Bruck, 1993). Ceci and Bruck (1993) explored the development of four primary aspects of cognition that influence suggestibility, starting with memory. The authors noted that the traces of a memory that younger children encode have been found to be weaker than those encoded by older children and adults. Further, younger children tend to encode memories in more details rather than using gist representations like adults (Ceci \& Bruck, 1993). Both of these factors of memory may cause the memories of younger children to degrade faster than those of older children and adults. Second, children's

less-developed linguistic skills may also lead to increased suggestibility. Ceci and Bruck (1993) stated that children have more problems understanding to-be-remembered events if they are presented verbally, and may have difficulty comprehending interview questions if they include complicated wording. Third, prior knowledge may influence suggestibility. As discussed by Ceci and Bruck (1993), younger children generally have less general knowledge than older children and adults which may negatively impact encoding and therefore increase suggestibility. On the other hand, the authors noted that less general knowledge may reduce the tendency for memory reconstruction errors. A final cognitive factor identified by Ceci and Bruck (1993) that influences suggestibility in children is source monitoring ability. This refers to children's ability to determine the correct source of knowledge. People must use source monitoring ability to 
distinguish between information that actually occurred in an event and information that did not occur but was suggested by some other source (Ceci \& Bruck, 1993; Ceci, Loftus, Leichtman, \& Bruck, 1994).

In a forensic setting, children may be suggestible; that is, they may incorporate information provided by the interviewer into their memories of the original event (Ceci \& Bruck, 1993; Ceci \& Friedman, 2000). Therefore, a way to reduce children's suggestibility is to improve their source monitoring ability (Ceci, et al., 1994). This is not a new idea and source monitoring ability has been a target of memory enhancement interventions for both adults and children (Lindsay \& Johnson, 1989). Although cognitive factors are noted above as reasons for age differences in suggestibility, it is important to point out that social (e.g., wanting to please the interviewer) and biological factors (e.g., stress) relate to suggestibility and may interact with cognition to influence suggestibility, although an in-depth discussion of these is beyond the scope of this document (Ceci \& Bruck, 1993; Goodman, Bottoms, Schwartz-Kenney, \& Rudy, 1991).

\section{Source Monitoring in Children}

Source monitoring is the cognitive task of tracking knowledge, including memories, back to their source (Johnson, Hashtroudi, \& Lindsay, 1993). An error in source monitoring occurs when the memory is attributed to a source that is not the actual source of the memory (Johnson, et al., 1993). For example, if I learned a particular piece of news from reading the paper, but I later thought I learned it from a conversation with a particular individual, this would be a source monitoring error.

Source monitoring ability changes over the course of development such that children have much different source monitoring capabilities compared to adults (Johnson, et al., 1993). 
Children have been found to have less accurate source memories than adults, with source monitoring ability improving throughout childhood into adulthood (Lindsay, Johnson, \& Kwon, 1991). Specifically, young children perform more poorly on a source monitoring task than older children (Drummey \& Newcombe, 2002; Lindsay et al., 1991; Welch-Ross, Diecidue, \& Miller, 1997). Developmental differences in source monitoring have been found in different types of source monitoring tasks. For example, these results have been found in studies utilizing more traditional source monitoring paradigms in which children receive information from two sources and must differentiate between the origins on a source memory test (Ackil \& Zaragoza, 1995; Bright-Paul, Jarrold, \& Wright, 2008). Similar differences in source monitoring ability between adults and children have also been found using a word list-learning task used to induce false recollections (Rybash \& Hrubi-Bopp, 2000).

The most significant development in source memory may occur early in childhood, during the preschool and early school-age years. In a cross-sectional study, Drummey and Newcombe (2002) examined source monitoring ability in 4-, 6-, and 8-year-old children. A notable improvement in source monitoring ability occurred between the 4- and 6-year-old groups, but there was no significant difference between the 6- and 8-year-old groups. Similarly, Lindsay et al. (1991) found a significant difference in source memory between 4- and 6-yearolds. These results suggest that the preschool and early school-age years may be an important period for the development of source memory.

Several researchers have hypothesized that the development of executive functioning in the prefrontal cortex may explain these age-related changes in source monitoring ability (BrightPaul et al., 2008; Roberts \& Powell, 2005; Ruffman, Rustin, Garnham, \& Parkin, 2001). Researchers have developed these hypotheses based on prior studies that established associations 
between frontal lobe dysfunction and source monitoring errors in adults (Craik, Morris, Morris, \& Loewen, 1990; Glisky, Polster, \& Routhieaux, 1995). Ruffman and colleagues (2001) explored this connection between source monitoring and executive functioning in children. An association was found between inhibition, an executive function, and source monitoring ability with greater inhibitory ability being involved with greater source monitoring accuracy. The authors suggested that being able to inhibit initial recognition based on familiarity or gist allowed children to make more accurate determinations of source.

Because the development of executive function skills has been associated with the emergence of theory-of-mind in children, it follows that theory-of-mind may also be related to source memory (Bright-Paul et al., 2008). Theory-of-mind refers to the "understanding of the representational nature of mind" or the understanding that each person experiences different mental states (Bright-Paul et al., 2008, pg. 1055). Bright-Paul and colleagues (2008) examined this relationship between theory-of-mind and source memory with children between the ages of 3 and 6. Specifically, the influence of the development of inhibitory control on theory-of-mind and source monitoring skills was explored. Children who had a greater theory-of-mind understanding were also more accurate on the source monitoring task, indicating that the development of both skills may be related. The authors suggest that improvements in source monitoring may explain the association between increased theory-of mind understanding and decreased suggestibility.

Individual differences in executive functioning skills may also impact source monitoring ability; these skills include inhibitory and attentional control. Roberts and Powell (2005) found that children who scored higher on a test of inhibitory control also performed more accurately on an eyewitness suggestibility task that required source monitoring skills to complete. Relatedly, 
evidence suggests that source monitoring ability may be related to children's attentional control abilities, which is distinct from but related to inhibitory control (Kanakogi, Moriguchi, Fu, Lee, \& Itakura, 2012).

Considering the important role the development of cognitive skills may play in the improvement of source memory (Johnson, et al., 1993), it can be expected that children experiencing certain cognitive deficits may also have reduced source monitoring abilities. In fact, source monitoring difficulties have been documented in children with disorders associated with cognitive impairment (Hill \& Russell, 2002; Lorsbach, Melendez, \& Carroll-Maher, 1991). Children with learning disabilities have been found to be less accurate on source monitoring tasks for verbal information than children without learning disabilities (Lorsbach \& Ewing, 1995; Lorsbach, et al., 1991). Further, children with fetal alcohol spectrum disorders were found to perform more poorly than control subjects on multiple source monitoring tasks (KullyMartens, Pei, Job, \& Rasmussen, 2012). The results for children with autism spectrum disorders are more mixed, with some studies indicating no significant differences in source monitoring between children with and without autism (Farrant, Blades, \& Boucher, 1998; Hill \& Russell, 2002). However, in some cases children with autism have been found to be less accurate on a source monitoring task than children without autism. These results were attributed to deficits in executive functioning (Hala, Rasmussen, \& Henderson, 2005).

\section{Source Monitoring and Suggestibility}

Source monitoring plays an important role in eyewitness testimony because source monitoring ability has been found to influence suggestibility in children (Ceci, et al., 1994). The introduction of false information following an event, or post-event misinformation, is associated with increased suggestibility in children and adults (Ayers \& Reder, 1998; Loftus, 1979; Loftus, 
et al., 1978). Situations that present post-event misinformation require the use of source monitoring to accurately recall what actually happened (Ayers \& Reder, 1998). Therefore, it makes sense that source monitoring ability may be related to suggestibility. Specifically, decreased source-monitoring ability has been associated with increased suggestibility in adults (Johnson et al., 1993). Orienting adults to the origins of their memories through a source monitoring task was found to decrease their suggestibility, highlighting the role of source memory in suggestibility (Lindsay \& Johnson, 1989).

A relationship between source-monitoring ability and suggestibility has also been established in children, and serves as one of the primary theories used to explain children's significant susceptibility to suggestive techniques (Johnson et al., 1993). Ceci et al. (1994) determined that children could be misled to believe that false events had actually happened to them, and conjectured that the mechanism driving these false memories was confusion about memory source. That is, children were unable to distinguish between personally experienced events and interviewer suggested events. In support of this hypothesis, Thierry, Spence, and Memon (2001) found that engaging children in source monitoring led them to have increased accuracy on a free recall task and less susceptibility to misleading questions. Similar studies have also found that children led through a source monitoring task are less suggestible during memory tests that follow (Bright-Paul, Jarrold, \& Wright, 2005; Giles, et al., 2002).

\section{Suggestibility Reduction via Source Monitoring Training}

The previously reviewed research indicates a well-established association between children's source monitoring accuracy and their accurate eyewitness testimony. Researchers have suggested that this association may serve as a point of intervention (Bruck, Melnyk, \& Ceci, 2000). In particular, increasing children's source monitoring accuracy may decrease their 
suggestibility (Thierry, et al., 2001). Several source monitoring training protocols have been designed and implemented to assess the efficacy of this intervention (Giles et al., 2002; Poole \& Lindsay, 2002; Thierry \& Spence, 2002). Training has been utilized to improve the source memory of adults as well (Engvig, Fjell, Westlye, Moberget, Sundseth, Larsen, \& Walhovd, 2010; Lane, Roussel, Villa, \& Morita, 2007; Martell \& Evans, 2005). The current study focused on source monitoring training protocols that have been designed for children in the context of suggestibility and eyewitness memory research.

In the first study of source monitoring training, preschool children were asked to distinguish between events they viewed on a video and events they simultaneously heard narrated (Giles et al., 2002). Thirty-two preschool children between the ages of 3 and 5 viewed a narrative story on a silent video and heard a spoken narrative of the story at the same time. Half of the children then engaged in a source monitoring task, where they were asked to determine the source of story details. This task served as the source monitoring training exercise and utilized the to-be-remembered event. The other half of the children participated in a control detail monitoring task also using the to-be-remembered event, where they were asked to recall story details. Children in each condition were also asked suggestive questions about the to-beremembered event. Finally, all children participated in a final suggestibility test regarding this to-be-remembered event. Children who received source monitoring training were better able to resist the influence of the suggestive questions as demonstrated by providing more accurate answers to the suggestibility test (Giles et al., 2002).

In a second study examining source monitoring training and suggestibility, children were asked to discriminate between an event that they saw live which later served as the to-beremembered event about which they were questioned and a similar event that they viewed on 
television (Thierry \& Spence, 2002). Thirty-six 3- and 4-year-old children viewed different science demonstrations live and through a video. In a second session, half of the children received source monitoring training. In contrast to the previously discussed training protocol, the training in this study was focused on events that the children experienced specifically for training purposes rather than the to-be-remembered events. Specifically, examples in the source monitoring training came from a separate event that all participants viewed live and on television regarding puppets. Children were asked a number of misleading questions about actions the puppets completed, and had to determine if these actions were completed live or in the video. The children were provided feedback regarding the accuracy of their answers. Children in the control condition were also asked misleading questions about the puppets' actions, but were not asked to recall source in their answers. Following the training conditions, children were asked open-ended and yes/no misleading and correctly leading questions about the live and televised to-be-remembered event.

The results of this study provided further support for the use of source-monitoring training with young children. Children in the source-monitoring training group were more accurate than children in the control group in response to misleading and correctly leading yes/no questions, and more accurate in response to open-ended questions about events that actually occurred (Thierry \& Spence, 2002). There was no significant difference between groups for open-ended questions about events that did not occur. These results provide evidence that source-monitoring training reduces suggestibility in 3- and 4-year-olds.

In a third study examining source monitoring training and suggestibility, Poole and Lindsay (2002) exposed 132 3-to-8-year-old children to live science demonstrations. They then had the children's parents read them books containing information that did and did not happen 
during the science demonstrations, which introduced misinformation to the children. Three months later, half of the children received a source-monitoring training procedure before a memory interview, while the other half of the children received the training after the memory interview. The source-monitoring training consisted of children watching a non-to-beremembered event performed by a researcher. Children were asked questions about the event, particularly which actions were actually performed by the researcher and which actions the researcher only talked about doing. They were given feedback regarding the accuracy of their answers. All children were then asked open-ended, misleading and correctly leading yes/no questions and source monitoring questions about the original live science demonstrations, but only the responses to the yes/no questions are relevant to this study.

The results of this study provide further evidence that training may reduce suggestibility, but imply that this effect may depend on the age of the children. Seven and eight-year-old children in the source monitoring condition reported fewer of the false, suggested details in response to misleading yes/no questions than children of the same age in the control condition (Poole \& Lindsay, 2002). However, there appeared to be no benefit of the source-monitoring training for 3- to 6-year-old children. Poole and Lindsay (2002) propose that younger children have difficulty generalizing the learning task from the source monitoring training to the source memory task. These conclusions contrast with other studies that have shown source monitoring effectively improves the accuracy of recall in young children between the ages of 3 and 6 years (Giles et al., 2002; Thierry, 2009; Thierry \& Spence, 2002).

In summary, two of the three previously described studies provide evidence that source monitoring training improves suggestibility for preschool children (Giles et al., 2002; Thierry \& Spence, 2002). However, source monitoring training is not the only technique that has been used 
to enhance the memory recall of children. One of these commonly used techniques implemented to improve children's eyewitness memory is the revised cognitive interview ${ }^{1}$ (Fisher \& Geiselman, 1992).

\section{The Cognitive Interview}

The cognitive interview is an interview protocol used to obtain information from eyewitnesses regarding their memories of the events they have experienced (Memon, Meissner, \& Fraser, 2010). Fisher and Geiselman (1992) created the revised cognitive interview based on the scientifically-established psychological principles of memory. Numerous studies have explored the efficacy of the interview and found that the protocol is able to significantly increase the number of correct details an individual recalls while only slightly enhancing the number of incorrect details recalled (for a review see Köhnken, Milne, Memon, \& Bull, 1999). The cognitive interview leads individuals through a detailed procedure to retrieve their memories of the original witnessed event (Memon et al., 2010).

The current version of the revised cognitive interview protocol consists partially of four different techniques that are utilized in a distinct sequence (as described by Fisher \& Geiselman, 1992). The first technique is context reinstatement, in which individuals are asked to mentally reconstruct the environment in which the original event occurred. The next technique is report everything, which instructs individuals to report everything they can remember without censoring their memories even if they do not believe that a particular detail will be relevant to solving the crime (i.e. even if they do not think the detail will be important). The third technique directs individuals to remember the event from a variety of perspectives, including their own

\footnotetext{
${ }^{1}$ Numerous different versions of the cognitive interview exist. When discussing relevant studies throughout this introduction, the interview will be referred to by the specific title that each author used in their study.
} 
viewpoints and the viewpoints of others. Finally, the last technique asks individuals to review the details of the witnessed event in various temporal orders, such as from the beginning to the end and reverse order recall from the end back to the beginning.

A recent meta-analysis reveals that the revised CI is effective in increasing adult's accurate recall $(d=1.20)$ with a small increase in incorrect recall $(d=0.24)$ (Memon et al., 2010). In children, the interview is associated with large increases in accurate details $(d=0.91)$ and smaller increases in inaccurate details recalled $(d=0.07)$. The effect sizes for these differences indicate that the cognitive interview does not improve recall as much in children as it does in adults. However, the cognitive interview also does not degrade recall accuracy, or increase incorrect recall, as much in children as it does in adults (Memon et al., 2010). Therefore, the cognitive interview has been deemed as an appropriate intervention for children to improve their recall of eyewitness events (Memon et al., 2010).

Although the revised cognitive interview may be used with children, some researchers have slightly modified the cognitive interview to be more developmentally appropriate (Holliday 2003a, 2003b; Milne \& Bull, 2003). A modified cognitive interview was devised for use with young children between the ages of 4 and 10 years (Holliday 2003a, 2003b). In this modified protocol all techniques of the cognitive interview were used except the step that asks witnesses to recall the event from multiple perspectives. This aspect of the cognitive interview was excluded because data indicated that young children were unable to complete this task, presumably due to children's reduced representational abilities (see also Saywitz, Geiselman, \& Bornstein, 1992). In both studies implementing this modified cognitive interview, children in the cognitive interview condition recalled more correct details about the witnessed event than children in the control condition (Holliday 2003a, 2003b). It should be noted that this effect of the interview 
was found when children provided free recall of their event memory, but not when they were asked specific yes/no questions about the event. This provides evidence that the modified cognitive interview may increase accurate free recall memory.

In addition to increasing accurate recall, there is some evidence that the cognitive interview may also reduce children's suggestibility during interview procedures (see Hayes and Delamothe, 1997; Holliday, 2003b for exceptions). In an examination of the modified cognitive interview developed by Holliday (2003a), children in the modified cognitive interview condition again reported more accurate details than children in the control condition. In contrast to Holliday's first examination of the modified cognitive interview (2003b), in this study the cognitive interview was associated with reduced suggestibility. Similarly, Milne and Bull (2003) found a suggestibility reduction effect using a cognitive interview protocol with children. The cognitive interview was associated with increased accurate open-ended recall and significantly decreased suggestibility. Related, but not identical, results were obtained by researchers using an original cognitive interview procedure (Memon, Holley, Wark, Bull, \& Köhnken, 1996). Interestingly, in the Memon et al. (1996) study there was no significant increase in recall of accurate information for children in the cognitive interview group compared to the control group. However, the cognitive interview was still found to reduce incorrect responses to suggestive questions.

In summary, research indicates that the cognitive interview is an effective memory enhancement technique that increases accurate recall in children and adults (Memon, et al., 2010). Although the cognitive interview also leads to some increase in erroneous recall, this effect is minimal compared to the much larger improvement in correct recall that is found when using this technique. There is also some evidence that the cognitive interview may decrease 
suggestibility in children, although further research is needed in this area given the discrepant results across studies (Holliday, 2003a; Memon, et al., 1996; Milne \& Bull, 2003). Taken together, these results imply that the cognitive interview is an appropriate memory enhancement technique to use with children that may lead to improvements in eyewitness memory (Fisher \& Schreiber, 2007).

\section{The Current Study}

The main goal of the current study was to examine the efficacy of source monitoring training in reducing suggestibility as compared to the most established memory enhancement technique that is used internationally in forensic interviews, the cognitive interview. The current study also included a control group, to control for practice effects of responding to yes/no questions and the effect of attention. Previous studies have shown that source monitoring training led to reduced suggestibility in children compared to a control condition (Giles et al., 2002; Thierry, 2009; Thierry \& Spence, 2002). Similarly, earlier research has shown that the cognitive interview is associated with increased recall in children compared to a control condition, and in some studies is associated with reduced suggestibility (Holliday, 2003a; Memon, et al., 2010; Milne \& Bull, 2003). However, the efficacy of source monitoring training and the cognitive interview have never been directly compared within the same study. This is an important comparison because the cognitive interview is currently used all over the world in forensic cases (Memon, et al., 2010). Therefore, it would be important to compare the most empirically-supported and widely used intervention to a newer intervention that demonstrates initial promise.

In the current study, children viewed a to-be-remembered event via video (Video 1). One week later, the children were randomly assigned to one of two memory enhancement techniques 
(source monitoring training or the cognitive interview) or a no-intervention control condition. Immediately following the source monitoring training or source monitoring control procedures, children received a memory interview to assess free recall and correct responses to suggestive questions. Free recall was obtained through the cognitive interview for children in the cognitive interview condition, while children in the other two conditions received an open-ended question. All participants then answered misleading and correctly leading suggestive questions.

Based on the results of prior studies, the following hypotheses were made:

Hypothesis 1: Source monitoring training is expected to be associated with higher misleading question accuracy compared to the no-intervention control group. Previous research has shown that individuals who receive source monitoring training are less susceptible to misleading questions compared to those in a control group who did not receive source monitoring training (Giles et al., 2002; Thierry, Lamb, Pipe, \& Spence, 2010; Thierry \& Spence, 2002).

Hypothesis 2: The cognitive interview is expected to be associated with a greater volume of accurate recall on the free recall task compared to the source monitoring training and nointervention control group. It is well-established that the cognitive interview can improve recall in children beyond a control group (Holliday 2003a, 2003b; Memon et al., 1996; Memon et al., 2010; Milne \& Bull, 2003). Therefore, participants in the cognitive interview condition are expected to perform better on free recall tasks than participants who have not received any memory enhancement intervention. Given that source monitoring training targets specific event details only, no significant differences are expected in free recall between the source monitoring training and control conditions. 
Hypothesis 3: Source monitoring training is expected to reduce suggestibility, which will be indicated by higher misleading question accuracy in the source monitoring training group compared to the control condition and the cognitive interview group. Some research indicates that individuals who are administered the cognitive interview may have reduced suggestibility (Holliday, 2003a, Memon et al., 1996, Milne \& Bull, 2003). A similar effect of source monitoring training on suggestibility has been found (Giles et al., 2002; Thierry \& Spence, 2002). The well-established relationship between children's source monitoring ability and suggestibility (Johnson et al., 1993) indicated that improving this ability through source monitoring training may reduce suggestibility more than the cognitive interview. This hypothesis will be examined for the first time in the current study.

In addition to the aforementioned specific hypotheses, the following two exploratory research questions were examined:

Research Question 1: Will there be differences in incorrect free recall between the three groups?

Research Question 2: Will there be differences between the three groups on correct responses to correctly leading questions?

\section{Method}

\section{Participants}

Participants were 37 children, 15 females and 22 males, ranging in age from 48 to 71 months $(M=59.65)$. Children were randomly assigned to experimental conditions, with 13 participants in the cognitive interview condition and 12 participants each in the source monitoring training and no-intervention control conditions. This cell size per condition equals or exceeds equivalent published studies that have found significant effects of source monitoring 
training and the cognitive interview (e.g., Giles et al., 2002; Holliday, 2003a). The mean age in months for children in each condition was 57.42 for the control condition, 60.83 for the source monitoring training condition, and 60.62 for the cognitive interview condition.

Demographic information was obtained through parent-report. Detailed information regarding these variables can be found in Table 1. Child participants were identified as $78.4 \%$ Caucasian, 8.1\% Biracial, 2.7\% African-American, 2.7\% Asian, and 2.7\% Hispanic/Latino; $5.4 \%$ of participants chose the "other" ethnic identification category. For $83.3 \%$ of participants, gross family income was indicated to be greater than $\$ 100,000$ per year. The primary caregivers of all participants reported the completion of a high school education. The majority of parents (94.5\%) earned additional post-secondary education degrees, with the majority of these parents earning a Master's degree (45.9\%).

Exclusion criteria stated that children could not have a diagnosis of autism spectrum disorder, learning disability, Attention-Deficit/Hyperactivity Disorder, or speech that is not clear enough to be understood on an audio recorder. These exclusion criteria were used because these diagnoses may directly influence children's memory abilities, as well as impacting their ability to follow instructions during the interventions (Hala et al., 2005; Lorsbach \& Ewing, 1995; Lorsbach et al., 1991).

Children were recruited from the Baltimore, MD and Washington, DC metro areas. Two primary methods of recruitment were used. First, advertising fliers were posted in community areas and on social media groups where children and families frequent. Second, participants were recruited from area daycares and schools. The study was advertised as a children's memory study. All recruitment materials and procedures were approved by the West Virginia University Institutional Review Board. The majority of enrolled participants were recruited 
through social media groups for parents and resulting word-of-mouth. Each family was paid \$20 for their child's participation in the study.

\section{Procedure}

Each participant participated in two study sessions scheduled one week apart. No longer than seven days elapsed between sessions. All sessions took place in the participant's home and were less than one hour in duration. The specific locations used in each home were chosen by the child's parent in collaboration with the researcher, with emphasis placed on minimizing extraneous noise and distractions. Although parents were permitted to observe within the same room if they chose to, they did not interact with their children throughout the study procedures. All recruitment was completed and all sessions were run by the primary researcher. All sessions were video-taped and memory interviews in the second sessions were also audio-recorded. The procedure for each session is detailed below. See Table 2 for a graphic depicting these procedures.

\section{Session 1.}

Upon the researcher's arrival at the participant's home, the child's primary caregiving parent completed the consent form and the demographic form. Children also assented to their participation in the study. Following consent and assent, children watched an approximately 10minute video (Video 1) of an adult male "toy inspector" inspecting five toys developed by Elisa Krackow, $\mathrm{PhD}$, which served as the to-be-remembered event. Children were not given any instructions to remember the events viewed in the video and were not given specific details about the procedure prior to or in the second session. 


\section{Session 2.}

In session 2, which occurred one week after session 1, either a memory enhancement intervention or a control procedure was implemented. Then all participants' recall of Video 1 (the to-be-remembered video) was assessed with a memory interview.

\section{Source monitoring procedures.}

Source monitoring training: source monitoring training condition.

Participants randomly assigned to the source monitoring condition participated in a source monitoring training developed by Elisa Krackow, $\mathrm{PhD}$, adapted from the training procedure created by Giles et al. (2002). After viewing the source monitoring training video (Video 2), children were asked eight question sequences about the video, with each sequence consisting of an event question and a source question. The first question was an event question consisting of a forced choice "yes/no" question about whether or not certain events occurred in the video. An example of an event question is, "Brian played with some blocks, didn't he?" All event questions were leading because they ended in “didn't he?" and thus suggested the event details contained in the question. Half of these questions were correctly leading (i.e. consistent with the actual events of the video), while the other half were misleading (i.e. inconsistent with the events of the video).

The second question in the sequence was a source question, a follow-up question about the source of the event detail. Specifically, children were asked if the event detail came from the video that they actually watched or from the question that they were currently being asked by the researcher. An example of a source question is, "Did I tell you Brian played with some blocks or did you see him play with some blocks in the movie?" Source questions encouraged the participants to consider and monitor the source of their memories when presented with leading 
questions and therefore served as source monitoring training. If children responded to either the event question or the source question incorrectly, their response was corrected and they were reasked that same question sequence (event plus source question) after all eight question sequences had been presented. It should be noted that it is not necessary to deliver additional specific instructions about source monitoring to children in order for them to utilize source monitoring training (Giles et al., 2002).

Source monitoring control procedure: cognitive interview and no-intervention control condition.

Participants randomly assigned to the cognitive interview or no-intervention control condition participated in a source monitoring control procedure developed by Elisa Krackow, $\mathrm{PhD}$. Participants watched the same source monitoring training video (Video 2) as the children in the source monitoring training condition. Following the video, they were asked the same "yes/no" questions about whether certain events occurred in the video (event questions) but were NOT asked if that detail came from the video or from the questions (source questions). That is, they were not asked to identify the source of their knowledge. This procedure served as a control for participants who did not receiving the source monitoring training procedure.

\section{Memory interviews.}

\section{Cognitive interview condition.}

Participants in the cognitive interview condition received the cognitive interview to obtain free recall. During the cognitive interview, children first received context reinstatement procedures (Step 1) by reinstating physical context. Specifically, both study sessions were conducted in the same location within the child's home to reinstate the context in which the original to-be-remembered video was viewed (Video 1). Next, children were asked an open- 
ended free recall question in which they were instructed to report everything they could recall even if they did not think it was important (Step 2: report everything). Children were asked specified prompts (i.e. “what else?” and “anything else?”) until they provided two consecutive denials of additional information. Following the report everything step, participants were asked to recall the event in the reverse order, starting with the detail they remembered that occurred last (Step 3: reverse order recall). Finally, they were asked to take the perspective of the "toy inspector" in the video and recall the events of the video from this perspective (Step 4: change perspectives). This version of the cognitive interview follows Holliday and Albon (2004) but was modified by Krackow.

Following the cognitive interview procedure, the children received 15 "yes/no" questions about the events of the video. These questions were also leading ( 5 correctly leading and 10 misleading) because they ended in “didn't he?" and suggested event details in the question. Therefore, these leading questions were used to assess suggestibility. The questions were developed for the video by Krackow for a different unpublished study. Previous pilot data has shown that children in the target age range are suggestible to these misleading questions.

\section{Source monitoring and no-intervention control conditions.}

In the source monitoring training and structured interview control conditions, second sessions occurred in different locations within the home than the first sessions to avoid the effects of physical context reinstatement given that context reinstatement is a component of the cognitive interview. Children in the source monitoring and structured interview control conditions received a standard interview which consisted of open-ended recall and leading "yes/no" questions. First, children were asked an open-ended free recall question in which they were instructed to report everything they could recall even if they didn't think it was important. 
This question was identical to the question used in the "report everything" step (Step 2) of the cognitive interview. In order to exhaust recall, children were asked specific prompts (i.e. "what else?" and “anything else?") until two consecutive denials of further information. Next, the children received 15 "yes/no" leading questions about the video. It should be noted that regardless of condition, all children received the same open-ended free recall question and the same "yes/no" leading questions.

\section{Debriefing.}

Following the memory test, child participants were thanked for their participation. They were debriefed via the researcher reading a written narrative (Krackow \& Lynn, 2010; Krackow \& Lynn, 2003). The researcher stated that she did not know what happened in the to-beremembered video (Video 1) so she asked questions about things that did and did not happen. The researcher reported that she "found" a paper left by the toy inspector explaining his actions, and the research read aloud the event components that did and did not happen when the toy inspector inspected the toys. Therefore, children completed the study with a clarified understanding of what occurred and what did not occur in the video. Parents were given the opportunity to ask any questions they had about the study procedures.

\section{Coding and Scoring.}

Memory reports were coded for their accuracy. Free recall memory reports were transcribed verbatim from audio-recordings of the interviews. A three-step process was used to code free recall reports. First, recall was broken into discrete ideas. Ideas were defined as the smallest spoken phrase that included a subject-verb pair and any accompanying objects, such as "fan was broken". Ideas may also consist of individual subjects or objects that were provided independent of an accompanying verb but could stand on their own to represent an idea. For 
example, "fish bowl". Filler statements were removed during this step and not coded, including "uhm", "I'm thinking", and "I don't know."

Next, each idea was coded as a complete or incomplete idea. A complete idea was described as including both a verb and a noun and could stand on its own as a sentence. An idea was defined as an incomplete idea if it did not constitute a sentence but rather contained a portion of a sentence, such as a subject or object.

Lastly, each complete or incomplete idea was coded as correct or incorrect. An idea was judged as correct if it accurately stated something that was viewed in the to-be-remembered video (Video 1). An idea was incorrect if it included anything that was not in the to-beremembered video (Video 1). The total number of correct ideas (i.e. complete correct ideas plus incomplete correct ideas) and the total number of incorrect ideas (i.e. complete incorrect ideas plus incomplete incorrect ideas) were then calculated. Complete ideas were worth one point and incomplete ideas were worth 0.5 points in this calculation. The total numbers of correct and incorrect ideas served as the dependent variables for the free recall memory task.

This coding system has been utilized in several memory studies to code free recall data and was developed by Elisa Krackow, PhD (e.g., Krackow Lynn, \& Payne, 2005). All free recall data was coded by one coder (the primary researcher), who had established reliability on $25 \%$ of the sample with the developer of the coding system and another trained graduate student. All coders were trained by the developer of the coding system. For complete/incomplete and correct/incorrect coding, reliability was established with complete agreement between the coders $($ Kappa $=1)$.

Leading "yes/no" questions that followed the free recall interview were scored for accuracy based on the actual events that occurred in the to-be-remembered video (Video 1). The 
number of correct answers was totaled and total accuracy scores were determined for both the misleading questions and the correctly leading questions. These total scores served as the dependent variables for the "yes/no" questions.

\section{Results}

The hypotheses and research questions of the study were explored using analyses of variance. Planned comparisons were used to assess specific effects delineated in the study hypotheses. To assess for pre-existing differences in suggestibility between groups, a univariate analysis of variance was performed on participants' responses to misleading questions (event questions) about Video 2 (the video in which Brian plays with toys). These questions were used because they were posed to participants in all three conditions prior to the implementation of the memory enhancement interventions. There was no effect of condition on accurate responses to misleading questions, $F(2,34)=0.431, p=.654$, indicating no differences in suggestibility between participants in the three conditions prior to the implementation of memory enhancement interventions.

The following outcome variables were analyzed in the study: total correct answers to misleading "yes/no" suggestibility questions $(M=6.108, S D=3.627)$, total correct answers to correctly leading "yes/no" suggestibility questions $(M=4.27, S D=.804)$, total number of correct ideas during free recall $(M=3.892, S D=4.44)$, and total number of incorrect ideas during free recall $(M=1.487, S D=2.712)$. One univariate outlier was found in the distribution of the variable assessing total number of correct ideas during free recall $(z$ score $=-4.98)$. Additionally, an examination of skewness and kurtosis z scores indicated that the skewness and kurtosis assumptions were violated for the total number of correct and incorrect free recall ideas. In order to correct the non-normality of these variables, a square root transformation was used to modify 
the scale of the distribution for the incorrect details variable. A log transformation was required for distribution of the correct details variable due to the severity of kurtosis. The transformed variables were used in all subsequent analyses.

\section{"Yes/no" Questions Accuracy: Misleading and Correctly Leading Questions}

To examine the effect of memory enhancement interventions on suggestibility, univariate analyses of variance were performed to clarify if there were differences in participants' correct responses to ten misleading "yes/no" questions. There was no effect of memory enhancement intervention on the number of correctly answered misleading questions across conditions, $F(2,34)=.096, p=.908$, partial $\eta^{2}=.006$. There were no significant differences in correct responses to misleading questions across the cognitive interview $(M=6.462, S D=3.332)$, source monitoring training $(M=6.00, S D=3.33)$, and control $(M=5.833, S D=4.428)$ conditions. Planned comparisons were used to examine the specific hypotheses that participants who received source monitoring training would respond more accurately to misleading questions compared to participants in the cognitive interview and no-intervention control conditions.

There was no significant effect of source monitoring training on increased accuracy to misleading questions compared to the no-intervention control condition $(t(34)=.11, p=.913, d$ $=0.04)$. Also, source monitoring training did not significantly increase accuracy to misleading questions compared to the cognitive interview $(t(34)=.31, p=.759, d=-0.14)$.

Similarly, there was no effect of memory enhancement intervention on participants' correct answers to five correctly leading questions across conditions, $F(2,34)=0.143, p=.867$, partial $\eta^{2}=.008$. There were no significant differences in correct answers to correctly leading questions across the cognitive interview $(M=4.307, S D=.63)$, source monitoring training $(M=$ 4.167, $S D=.835)$, and control $(M=4.333, S D=.985)$ conditions. Planned comparisons yielded 
no significant effect of the source monitoring training condition on increased accuracy to correctly leading questions compared to the no-intervention control condition $(t(34)=-.495, p=$ $.624, d=-0.18)$, nor in comparison to the cognitive interview condition $(t(34)=.427, p=.672, d$ $=-0.19)$.

Two participants in the source monitoring training condition were considered unresponsive to training because they continued to respond inaccurately to source questions during the source monitoring training. When the data from these individuals was removed from the analyses, there continued to be no effect of memory enhancement intervention on participants' correct answers to misleading questions $\left(F(2,32)=0.424, p=.658\right.$, partial $\eta^{2}=$ $.026)$ and correctly leading questions $\left(F(2,32)=0.251, p=.78\right.$, partial $\left.\eta^{2}=.015\right)$. The mean number of correct answers to misleading questions increased in the source monitoring condition from $6.0(S D=3.33)$ to $7.2(S D=1.989)$, but this increase did not change the results. Specifically, source monitoring training did not increase accurate responses to misleading questions compared to the control $(t(32)=.921, p=.364, d=0.40)$ or cognitive interview $(t(32)$ $=-.506, p=.616, d=0.27$ ) conditions. Source monitoring training also did not increase accurate responses to correctly leading questions compared to the control $(t(32)=-.652, p=.519, d=-$ $0.25)$ and cognitive interview $(t(32)=.591, p=.559, d=-0.27)$ conditions.

\section{Free Recall Memory Accuracy: Correct and Incorrect Recall}

To assess the effect of memory enhancement interventions on the accuracy of participants' free recall, univariate analyses of variance examined differences between conditions on total number of correct and incorrect free recall ideas. It should be noted that three participants (one in the control condition and two in the source monitoring training condition) did not provide any ideas that could be coded as correct or incorrect. There was no effect of 
memory enhancement intervention on total number of correct ideas, $F(2,34)=1.232, p=.304$, partial $\eta^{2}=.068$. There was no evidence that there were a greater number of correct ideas in the cognitive interview condition $(M=5.269, S D=6.663)$ or the source monitoring training condition $(M=2.458, S D=1.544)$ compared to the control condition $(M=3.833, S D=3.033)$. Planned comparisons indicated no significant effect of the cognitive interview on accurate free recall compared to the no-intervention control condition $(t(34)=.585, p=.562, d=0.28)$.

An analysis of variance was also performed to clarify if there were differences in number of incorrect ideas reported during free recall across the three conditions. There was no statistically significant effect of memory enhancement interventions on total number of incorrect ideas but the effect size was large, $F(2,34)=2.897, p=.069$, partial $\eta^{2}=.146$. Participants in the cognitive interview condition $(M=2.808, S D=3.437)$ and the source monitoring training condition $(M=0.583, S D=1.165)$ were not found to recall significantly more incorrect ideas than participants in the control condition $(M=0.958, S D=2.562)$. Planned comparisons reported that the effect of the cognitive interview on inaccurate free recall as compared to the nointervention control condition approached but did not reach significance $(t(34)=1.989, p=$ $.055)$. However, an examination of effect size indicated moderate effects $(d=0.61)$ of the cognitive interview on increasing inaccurate free recall as compared to the control condition.

To further explore the influence of the cognitive interview, accurate and inaccurate free recall at each component of the cognitive interview was calculated for all participants in this condition. In the report everything component (step 2), the participants were found to report a mean of 3.654 correct ideas $(S D=5.854)$ and a mean of .769 incorrect idea units $(S D=1.3)$. In the reverse-order recall step (step 3), a mean of .962 additional correct ideas $(S D=1.233)$ and a mean of .231 additional incorrect ideas $(S D=.439)$ were found. Finally, in the change 
perspectives step (step 4), participants were found to report a mean of .654 additional correct ideas $(S D=1.162)$ and a mean of 1.81 additional incorrect ideas $(S D=2.689)$.

Throughout the study, anecdotal evidence from the primary researcher indicated that participants may have had particular difficulty with the change perspectives component of the cognitive interview. Saywitz and colleagues (1992) previously suggested similar concerns with this component of the interview. Therefore, correct and incorrect ideas from this step of the interview were removed for participants in the cognitive interview condition and the data were re-analyzed. However, there continued to be no effect of memory enhancement intervention on total number of correct ideas $\left(F(2,34)=.606, p=.551\right.$, partial $\left.\eta^{2}=.03\right)$ or total number of incorrect ideas $\left(F(2,34)=.145, p=.865\right.$, partial $\left.\eta^{2}=.008\right)$. Specifically, planned comparisons continued to indicate no significant effect of the cognitive interview on correct $(t(34)=.006, p=$ $.995, d=0.15)$ and incorrect $(t(34)=.383, p=.704, d=0.02)$ free recall as compared to the nointervention control condition.

\section{Discussion}

The current study explored the impact of two memory enhancement techniques on free recall and suggestibility in young children. Specifically, the study examined the effects of source monitoring training and the cognitive interview on open-ended recall and accurate responses to misleading and correctly leading questions. It was hypothesized that source monitoring training would reduce suggestibility to a greater extent than the cognitive interview or a no-intervention control, as evidenced by very large effect sizes in previous studies and more consistent results across studies than the cognitive interview. It was also hypothesized that the cognitive interview would elicit increased accurate free recall compared to an open-ended memory interview in the source monitoring training and no-intervention control conditions. However, the results of the 
current study do not support these hypotheses. These results are contrary to previous research that found positive effects of these memory enhancement interventions on free recall and suggestibility in children.

\section{Suggestibility}

The source monitoring training intervention was not found to increase accuracy compared to the no-intervention control condition on a suggestibility task. Prior research has indicated that improved source monitoring accuracy is associated with reduced suggestibility in children and adults (Johnson et al., 1993). Source monitoring training was developed to increase source monitoring accuracy and has been found to reduce susceptibility to suggestive questioning techniques in previous studies with children (Giles et al., 2002; Thierry \& Spence, 2002). This memory enhancement technique was predicted to have a similar result in the current study when compared to a control group that did not receive source monitoring practice. The results suggest that source monitoring training may not be sufficient to reduce suggestibility, at least when this specific protocol of source monitoring training is used.

It should be noted that the cognitive interview condition was not found to reduce suggestibility beyond the effects of a no-intervention control, which is contrary to a previous finding that cognitive interview may lead to some decrease in suggestibility (Holliday, 2003a; Memon et al. 1996; Milne \& Bull, 2003). In fact, participants' high rates of suggestibility across conditions in response to single presentations of leading questions are consistent with participants' suggestibility in control conditions from previous studies (Krackow \& Lynn, 2003, 2010). This indicates that neither intervention in the current study was found to reduce suggestibility, which is contrary to prior research and suggests the possibility that other factors may be impacting these results. 
All participants were included in the analyses that examined the effect of source monitoring training on suggestibility, including participants who did not fully comprehend the training. To further explore these results, the effectiveness of the source monitoring training was considered. Participants who did not respond to the intervention, as evidenced by continued inaccurate responses to source questions following feedback and repetition, were removed from the sample. Those that did not respond to the intervention would not be expected to exhibit the predicted effect of reduced suggestibility. When the data from the two participants who did not comprehend the source monitoring training were removed from the analyses, the mean number of correctly answered misleading questions increased for the source monitoring training group. However, the results remained the same and source monitoring training did not reduce suggestibility compared to the cognitive interview and no-intervention control conditions. This indicates that the lack of effect of source monitoring training was not due to the inclusion of participants who did not respond to the intervention.

Considering the impact of development on source monitoring ability, it could be expected that some children in the current sample were found to not fully comprehend and respond to the source monitoring training. Specific cognitive skills that develop throughout childhood have been associated with improved source monitoring ability (Bright-Paul et al., 2008; Roberts \& Powell, 2005; Ruffman et al., 2001). It follows that individual cognitive development may influence the efficacy of source monitoring training as children develop these cognitive skills at differing rates, causing some children to not understand the procedure and therefore not benefit from the intervention. In the current study, difficulty comprehending the source monitoring training in a small portion of the sample did not explain the unexpected lack of effect for the source monitoring training intervention. 
The nonsignificant results of the current study add to existing mixed evidence for the effect of source monitoring training on suggestibility, thereby continuing to raise questions regarding whether source monitoring training is effective for preschoolers. Two studies have found that training increased accuracy for suggestive questions in children aged 3 to 5 years (Giles et al., 2002; Thierry \& Spence, 2002). Poole and Lindsay (2002) did not find this effect for participants between 3 and 6 years of age, nor was the effect significant in the current study.

Differences in the source monitoring training procedure may explain the lack of significant results in the current study. In the studies that found a positive effect of source monitoring training, misleading information was introduced live or on video. Children were then asked source monitoring questions that involved identifying if specific details occurred live or in video. In the studies that did not find a positive effect of source monitoring training, including the current study, misleading information was introduced as part of the source monitoring questions. That is, the child was asked if a specific detail actually happened or if the interviewer just talked about it happening. This suggests that other source monitoring training protocols may be found to improve eyewitness memory with this population. Taken together, the current study and previous literature indicate that source monitoring training cannot be reliably used as a memory enhancement technique in forensic settings until further research determines the efficacy of this intervention with young children.

Additionally, the source monitoring training intervention did not increase accuracy on a suggestibility task as compared to the cognitive interview condition. Although the cognitive interview has been found to reduce suggestibility in some previous studies (Holliday, 2003a, Memon et al., 1996, Milne \& Bull, 2003), source monitoring training was expected to reduce suggestibility above and beyond this effect because of its specific impact on source monitoring 
ability. As previously discussed, there is a well-established link between source monitoring ability and accuracy in response to suggestive techniques. Source monitoring training and the cognitive interview were compared directly as memory enhancement techniques, a comparison that has not occurred in prior literature, with the results suggesting that source monitoring training does not provide an additional benefit to reduce suggestibility beyond the effect of the cognitive interview.

Compared to distinguishing between two viewed events, young children may find it particularly difficult to differentiate between an event they viewed and an event that was solely talked about (by the interviewer). Source monitoring training protocols that introduce misleading information as part of the source monitoring questions may still be effective when used with older children who are better able to make this differentiation. For example, Poole and Lindsay (2002) found that their source monitoring training protocol was associated with reduced suggestibility in their older sample of 7- and 8-year-old children. Taken together, previous research and the results of the current study indicate that source monitoring training may be an effective intervention to reduce suggestibility, but young children may require primarily visual rather than verbal training materials to induce this effect.

\section{Free Recall}

The cognitive interview condition was not found to increase accurate free recall compared to the source monitoring and no-intervention control conditions. This memory enhancement technique was predicted to increase recall compared to a no-intervention control because multiple studies with children have shown that it provides greater accurate recall than control groups (Holliday 2003a, 2003b; Memon et al., 1996; Memon et al., 2010; Milne \& Bull, 2003). A similar effect was expected in the current study because a similar cognitive interview 
format on a free recall task was used. The cognitive interview was also predicted to elicit greater free recall compared to the source monitoring training condition because the source monitoring training condition focuses on specific event details rather than global recall of the event. The results of the current study indicate that the cognitive interview did not elicit greater accurate recall compared to two conditions that lacked any memory enhancement intervention during the memory interview.

This finding is unexpected as it does not align with prior literature. A large body of previous research has shown that the cognitive interview significantly increases recall of correct details while slightly increasing recall of incorrect details (Köhnken et al., 1999; Memon et al., 2010). This effect has been established in adults and children, leading interviewers to use the cognitive interview widely in eyewitness memory situations. The cognitive interview has been found to be particularly effective in obtaining free recall from children (Holliday 2003a, 2003b), as was assessed in the current study. The results for inaccurate free recall showed a trend that aligns with these widely known effects of the cognitive interview. A nonsignificant but moderate effect of the cognitive interview on inaccurate free recall was found, indicating that participants who received the cognitive interview provided slightly more incorrect details compared to the other two groups. This indicates that the cognitive interview may have had some impact on free recall in the current study in the expected direction for inaccurate memory. The nonsignificance of this moderate effect may be an issue of low power due to sample size, suggesting that this result may have been significant if the sample size was increased.

The concern about power due to small sample size does not generalize to the findings regarding the effect of the cognitive interview on accurate free recall. The cell size for each condition in the current study equaled those in a similar study that found a significant effect of 
the cognitive interview on free recall in children (Holliday, 2003a). The effect sizes for the impact of the cognitive interview on accurate and inaccurate free recall were also large in prior research, in comparison to the small effect found for accurate recall in the current study. Therefore, the effect of the cognitive interview appears to be considerably reduced in the current study and may not have been significant even within a large sample. Given that an increase in accurate recall would have the most meaningful influence on improving eyewitness memory, the results suggest that the cognitive interview would not have had clinically significant utility in the current study even with a large sample.

Considering that the effects of the cognitive interview on children's memory have been well-established in the literature, the lack of significant results in the current study may be impacted by the specific cognitive interview protocol used. During Step 1 of the interview, children received physical rather than mental context reinstatement as the interview was completed in the same room in which the to-be-remembered video (Video 1) was viewed. Mental reinstatement of physical context is most often used in cognitive interviews. In the current study, physical reinstatement was chosen because the youngest children in the sample (i.e. 4-year-olds) were expected to have reduced abilities to mentally reinstate context due to their stage of cognitive development compared to the older children and adults that have served as participants in previous studies of the cognitive interview. However, using the same nondistinct physical location may have not sufficiently reinstated the context of the to-beremembered event. A previous comparison of physical and mental context reinstatement in children found that both conditions elicited comparable amounts of information but greater detail and quality of information was obtained in the mental context reinstatement condition (Hershkowitz, Orbach, Lamb, Sternberg, \& Horowitz, 2002). The current study was originally 
designed to take place in a laboratory setting, which would have allowed participants to experience the to-be-remembered event (Video 1) and the cognitive interview in the same unique, never before encountered location. After the procedure was adjusted to take place in each child's home, the cognitive interview took place in a location the child was familiar with and likely spent time in during the one week lapse between the to-be-remembered event and the memory interview. This may have furthered reduced the efficacy of physical context reinstatement.

The cognitive interview protocol also utilized the change perspectives technique (Step 4) which asked participants to take the perspective of the toy inspector in the to-be-remembered video (Video 1). This step was utilized due to its inclusion in the modified cognitive interview commonly used in eyewitness settings. However, it has previously been noted that children may have difficulty understanding and utilizing this instruction due to developmental difficulties with perspective-taking (Saywitz, et al., 1992). This technique has been removed from some cognitive interview protocols modified for children because of these concerns (Holliday 2003a, 2003b; Milne \& Bull, 2003). In the current study, the researcher observed that a number of participants did not appear to understand the change perspective instruction. They interpreted this question as the researcher asking the participants what toys they would play with if they were a toy inspector. Further verbal explanation from the researcher did not successfully clarify the instruction. To determine if this misunderstanding reduced the effect of the cognitive interview on free recall, the sample was re-analyzed with all data from the "change perspectives" step removed for all participants in the cognitive interview condition. The results remained unchanged and there was still no effect of the cognitive interview on accurate and inaccurate free recall. 
An element of the research design that may have impacted the efficacy of the cognitive interview was the one-week delay between the viewing of the to-be-remembered event (Video 1) in session 1 and the implementation of the cognitive interview in session 2. Previous studies of the cognitive interview in children and adults have typically used shorter delays of one to three days, especially in studies that utilized video presentation of the to-be-remembered event (Kohnken, et al., 1999; Memon, 2010). For example, several studies that found the cognitive interview to be effective in improving recall in young children between the ages of 4 and 10 utilized video presentation and short delays (Hayes \& Delamothe, 1997; Holliday, 2003a; 2003b; Milne \& Bull, 2003). A week delay was chosen in the current study because previous pilot studies and a study by Krackow and Lynn (2003) found that this delay was ideal to obtain an effect of memory interventions on suggestibility. However, this longer delay may have led free recall memory to decay too much, leading to forgetting. Memory that has been forgotten cannot be improved by interventions and this may have rendered the cognitive interview ineffective.

Another aspect of the research design that may have impacted the efficacy of the cognitive interview was the use of a single researcher to run all steps of the experiment. The study was originally designed with three different researchers implementing each step in the experiment: a) viewing of the to-be-remembered video (Video 1), b) viewing of the source monitoring video (Video 2) and subsequent questions, and c) the memory interviews. This design was revised to allow for implementation of the experiment in participants' homes in order to increase accessibility, and it became necessary for all steps of the experiment to be implemented by the same researcher for feasibility. This choice may have negatively impacted the results of the study because participants may have assumed that the researcher had some knowledge of the to-be-remembered event (Video 1) because the researcher was involved in 
presenting the video. This may have led the children to exhibit reduced recall during the memory interview because they made inferences about what the researcher already knew and did not provide all recalled details based on these assumptions, even though children were told to include all details they recalled. Overall reduced recall may have negatively impacted the effect of the cognitive interview on free recall. These results suggest that including additional personnel to implement study procedures may an appropriate and effective use of resources because it may lead to more robust results in experiments that examine free recall.

\section{Research Design}

The current study utilized a unique research design in which sessions were conducted within participants' homes. This method allowed the primary researcher to collect a large amount of data independently. Experimental control was reduced because the researcher had little influence over the larger setting in which the sessions took place. Extraneous situational factors were occasionally present in the form of noise, visual distractions, and the presence of other people, even though all research equipment and procedures were the same for every participant. These served as threats to the reliability and internal validity of the study design.

Despite these concerns about in-home research, studies conducted outside of the laboratory setting may benefit from increased ecological validity and applicability. Children are most likely to experience real-life to-be-remembered events in their homes and similar uncontrolled settings rather than in the laboratory where many memory studies occur. Further, this design may be more acceptable to families, as compared to entering an unfamiliar laboratory setting. Removing the burden of attending sessions from families may allow researchers to recruit a more diverse sample, in terms of demographic and geographic variables. Bringing the researcher to the participant may also reduce common recruitment concerns, including canceled 
and no showed sessions. In some cases, the financial burden of conducting research may be reduced if researchers are not required to maintain a physical research laboratory.

Although the current study provided evidence that it is possible to conduct a memory study in participants' homes, the memory enhancement interventions utilized in the study were not found to be effective in reducing suggestibility and improving recall. As previously discussed, these results were contrary to extensive prior literature indicating the efficacy of these interventions. The lack of significant results in the current study may be due to conducting the memory interviews in the participants' homes, which differs from traditional best practice procedures in forensic interviewing. It is a widely supported assertion in forensic literature that eyewitness memory interviews should not be conducted in children's homes (Cross, Jones, Walsh, Simone, \& Kolko, 2007). Prior research has established the importance of completing eyewitness memory interviews in controlled settings to improve accurate recall, and interviewing children in Child Advocacy Centers or interviewing centers has been determined as best practice (APSAC, 2002; Cross, et al., 2007). The current study provides additional evidence that eyewitness memory and forensic interviews should not be conducted in children's homes, particularly because two memory enhancement interventions with strong empirical support were not effective in this setting. The results of this study can be used to justify the importance of maintaining appropriate interviewing centers for children that can be used in combination with memory enhancement techniques to increase interviewers' chances of obtaining accurate recall.

However, the efficacy of source monitoring training and the cognitive interview implemented in-home cannot be directly compared with their efficacy in laboratory settings because the current study was only conducted in one setting. The results of the current study also cannot be compared to previous research because there are no prior studies that examine the 
use of these interventions in participants' homes. Consequently, it cannot be determined if the lack of significant results in the current study is due to the experimental setting. Future research should compare the outcomes of these interventions in multiple settings. An experiment that randomizes participants to receive these interventions in the laboratory or their homes would allow researchers to asses for the effects of setting. Researchers would be able to further determine the impact of obtaining eyewitness memories in children's homes, which could inform guidelines for forensic interviewing procedures.

\section{Future Directions}

Prior research has shown that source monitoring training and the cognitive interview may be effective interventions to improve children's eyewitness memory. These techniques were not effective in reducing suggestibility and increasing accurate recall in the current study. Examination of these results has identified that specific factors of the interventions may have significantly impact their efficacy. Future research should continue to examine the use of these memory enhancement techniques with the incorporation of the factors discussed in this document.

In exploring the mixed evidence supporting the use of source monitoring training to reduce suggestibility, it was found that the method through which misleading information is introduced may impact the efficacy of the intervention, especially for young children. Further studies should examine differences in children's ability to monitor the source of information viewed visually, as compared to information that is viewed and verbally delivered. The most effective protocol for source monitoring training may vary for children based on age, due to large changes in cognitive abilities across childhood. 
The research design in the current study introduced additional opportunities for error because completing sessions in-home reduces experimental control. Therefore, the hypotheses of the current study should continue to be explored in a laboratory setting to determine if the lack of significant results is due to this unique design. However, the successful completion of this study indicates that in-home experimental research is feasible. The majority of studies exploring children's memory enhancement have occurred in a traditional laboratory setting or structured community setting, such as a school. The current study introduces the use of the home as a research setting. Many possible benefits associated with this form of research have been discussed. The field would benefit from further exploration of the use of in-home research as compared to the traditional in-lab design. Completing experimental studies in homes and community settings may allow researchers to reduce costs, increase efficiency, and improve the real-world applicability of their interventions.

Moving experimental studies outside of the laboratory also opens up diverse recruitment and research design opportunities. A relatively diverse sample of participants was recruited in the current study, including female and male children spanning the preschool age range (i.e., 48 to 71 months of age) from a variety of ethnic backgrounds. This sample of preschool children represents the age of children that may most benefit from effective interviewing techniques in forensic situations. However, despite using a number of recruitment techniques over a wide geographic area, relatively all families who participated in the study reported above-average household income and parental education levels. The middle to high socioeconomic status of this sample is a limitation of the study because it does not represent the population of children most likely to receive forensic interviews. Eyewitness testimony is most often sought from children of low-SES because they have a higher probability of experiencing abuse (Dorado \& 
Saywitz, 2001). Future research would benefit from the incorporation of children from low-SES backgrounds to ensure that the memory enhancement techniques will be effective for the children who are most likely to receive them as part of a forensic interview.

\section{Conclusions}

The current study examined the effect of two memory enhancement techniques on children's eyewitness memory. Neither source monitoring training nor the cognitive interview were found to reduce suggestibility or improve free recall compared to a no-intervention control condition. Specifically, source monitoring training did not lead to greater accuracy on misleading questions compared to the cognitive interview or control conditions. The cognitive interview also did not produce greater accurate free recall than source monitoring training or the control condition. Therefore, the proposed study hypotheses were not supported.

The results of the current study add to the existing mixed evidence regarding the efficacy of source monitoring training as an intervention for suggestibility in preschool children. Because early childhood appears be an imperative age for the development of source monitoring abilities (Drummey \& Newcombe, 2002; Lindsay et al., 1991), preschool children may respond to source monitoring training differently based on individual differences in cognitive development. The nonsignificant results in the current study suggest that children may benefit more from some training protocols than others. For example, as previously discussed, young children may benefit more from live or video presentation of misleading information rather than presenting this information as part of the source monitoring questions as in the current study.

The lack of significant results in the current study does not align with extensive prior literature indicating that the cognitive interview is an effective intervention to improve eyewitness memory in children. Several aspects of the cognitive interview protocol used in the 
current study may have influenced the efficacy of the intervention. Specific steps used in the interview, such as the physical context reinstatement and change perspectives steps, may be developmentally inappropriate for preschool children and yield minimal effect (Hershkowitz, et al., 2002; Saywitz, et al., 1992). Other aspects of the study design may have reduced the effects of the intervention, including the use of a single researcher to run all components of the experiment and the use of a one-week delay between the to-be-remembered event and the memory interview. Many different versions of the cognitive interview exist and few studies have directly compared the efficacy of different interview elements in children. Therefore, the current study provides some insight into aspects of the interview that may reduce the efficacy of this intervention.

Conducting the current study in participants' homes was a marked departure from traditional eyewitness memory research that is carried out in highly controlled laboratory settings. There are a number of benefits associated with in-home research, including greater ecological validity and applicability to real-life forensic situations. Conversely, the reduced experimental control in this setting may reduce the efficacy of the interventions. Although substantial effort was dedicated to maintaining experimental control in participants' homes, the absence of significant results in the current study may have been influenced by the experimental setting. The current study consequently provides support for current best practice forensic interviewing procedures that state interviews should occur in interviewing centers rather than children's homes (APSAC, 2002; Cross, et al., 2007). 


\section{References}

Ackil, J. K., \& Zaragoza, M. S. (1995). Developmental differences in eyewitness suggestibility and memory for source. Journal of Experimental Child Psychology, 60, 57-83. doi:10.1006/jecp.1995.1031

American Professional Society on the Abuse of Children. (2002). Investigative interviewing in cases of alleged child abuse. Charleston, SC: APSAC. Practice Guidelines Series.

Ayers, M. S., \& Reder, L. M. (1998). A theoretical review of the misinformation effect: Predictions from an activation-based memory model. Psychonomic Bulletin \& Review, 5, 1-21. doi:10.3758/BF03209454

Bright-Paul, A., Jarrold, C., \& Wright, D. B. (2005). Age-appropriate cues facilitate sourcemonitoring and reduce suggestibility in 3- to 7-year-olds. Cognitive Development, 20, 118. doi:10.1016/j.cogdev.2004.06.001

Bright-Paul, A., Jarrold, C., \& Wright, D. B. (2008). Theory-of-mind development influences suggestibility and source monitoring. Developmental Psychology, 44, 1055-1068. doi:10.1037/0012-1649.44.4.1055

Bruck, M., Melnyk, L., \& Ceci, S. J. (2000). Draw it again Sam: The effect of drawing on children's suggestibility and source monitoring ability. Journal of Experimental Child Psychology, 77, $169-196$.

Ceci, S. J., \& Bruck, M. (1993). Suggestibility of the child witness: A historical review and synthesis. Psychological Bulletin, 113, 403-439. doi:10.1037/0033-2909.113.3.403

Ceci, S. J., \& Friedman, R. D. (2000). The suggestibility of children: Scientific research and legal implications. Cornell Law Review, 86, 34 - 108. 
Ceci, S. J., Loftus, E. F., Leichtman, M. D., \& Bruck, M. (1994). The possible role of source misattributions in the creation of false beliefs among preschoolers. International Journal of Clinical and Experimental Hypnosis, 42, 304-320. doi:10.1080/00207149408409361

Craik, F. I., Morris, L. W., Morris, R. G., \& Loewen, E. (1990). Relations between source amnesia and frontal lobe functioning in older adults. Psychology and Aging, 5, 148-151. doi:10.1037/0882-7974.5.1.148

Cross, T. P., Jones, L. M., Walsh, W. A., Simone, M., Kolko, D. (2007). Child forensic interviewing in Children's Advocacy Centers: Empirical data on a practice model. Child Abuse \& Neglect, 31, 1031-1052.

Dorado, J. S., \& Saywitz, K. J. (2001). Interviewing preschoolers from low- and middle-SES communities: A test of the narrative elaboration recall improvement technique. Journal of Clinical Child Psychology, 30, 568-580.

Drummey, A. B., \& Newcombe, N. S. (2002). Developmental changes in source memory. Developmental Science, 5, 502-513. doi:10.1111/1467-7687.00243

Engvig, A., Fjell, A. M., Westlye, L. T., Moberget, T., Sundseth, Ø., Larsen, V., \& Walhovd, K. B. (2010). Effects of memory training on cortical thickness in the elderly. Neuroimage, 52, 1667-1676. doi:10.1016/j.neuroimage.2010.05.041

Farrant, A., Blades, M., \& Boucher, J. (1998). Source monitoring by children with autism. Journal of Autism and Developmental Disorders, 28, 43-50. doi:10.1023/A:1026010919219

Fisher, R. P., \& Geiselman, R. (1992). Memory-enhancing techniques for investigative interviewing: The cognitive interview. Springfield, IL England: Charles C Thomas, Publisher. 
Fisher, R. P., \& Schreiber, N. (2007). Interviewing protocols to improve eyewitness memory. The Handbook of Eyewitness Psychology, 1, 53-80.

Geiselman, E. R., Fisher, R. P., MacKinnon, D. P., \& Holland, H. L. (1985). Eyewitness memory enhancement in the police interview: Cognitive retrieval mnemonics versus hypnosis. Journal of Applied Psychology, 70, 401-412.

Geiselman, E. R., Fisher, R. P., MacKinnon, D. P., Holland, H. L. (1986). Enhancement of eyewitness memory with the cognitive interview. The American Journal of Psychology, 99, 385-401.

Giles, J. W., Gopnik, A., \& Heyman, G. D. (2002). Source monitoring reduces the suggestibility of preschool children. Psychological Science, 13, 288-291. doi:10.1111/1467-9280.00453

Glisky, E. L., Polster, M. R., \& Routhieaux, B. C. (1995). Double dissociation between item and source memory. Neuropsychology, 9, 229-235. doi:10.1037/0894-4105.9.2.229

Goodman, G.S., Bottoms, B.L., Schwartz-Kenney, B.M., \& Rudy, L. 1991. Children's testimony about a stressful event. Journal of Narrative and Life History, 7, 69-99.

Hala, S., Rasmussen, C., \& Henderson, A. E. (2005). Three Types of Source Monitoring by Children With and Without Autism: The Role of Executive Function. Journal of Autism and Developmental Disorders, 35, 75-89. doi:10.1007/s10803-004-1036-4

Hayes, B. K., \& Delamothe, K. (1997). Cognitive interviewing procedures and suggestibility in children's recall. Journal of Applied Psychology, 82, 562-577. doi:10.1037/00219010.82 .4 .562

Hershkowitz, I., Orbach, Y., Lamb, M. E., Sternberg, K. J., \& Horowitz, D. (2002). A comparison of mental and physical context reinstatement in forensic interviews with alleged victims of sexual abuse. Applied Cognitive Psychology, 16, 429-441. 
Hill, E. L., \& Russell, J. (2002). Action memory and self-monitoring in children with autism: Self versus other. Infant and Child Development, 11, 159-170. doi:10.1002/icd.303

Holliday, R. E. (2003a). Reducing misinformation effects in children with cognitive interviews: Dissociating recollection and familiarity. Child Development, 74, 728-751. doi:10.1111/1467-8624.00565

Holliday, R. E. (2003b). The effect of a prior cognitive interview on children's acceptance of misinformation. Applied Cognitive Psychology, 17, 443-457. doi:10.1002/acp.879

Holliday, R. E., \& Albon, A. J. (2004). Minimising misinformation effects in young children with cognitive interview mnemonics. Applied Cognitive Psychology, 18, 263-281. doi:10.1002/acp.973

Johnson, M. K., Hashtroudi, S., \& Lindsay, D. (1993). Source monitoring. Psychological Bulletin, 114, 3-28. doi:10.1037/0033-2909.114.1.3

Kanakogi, Y., Moriguchi, Y., Fu, G., Lee, K., \& Itakura, S. (2012). How does executive function contribute to source monitoring in young children? Psychologia: An International Journal of Psychological Sciences, 55, 194-207. doi:10.2117/psysoc.2012.194

Köhnken, G., Milne, R., Memon, A., \& Bull, R. (1999). The cognitive interview: A metaanalysis. Psychology, Crime \& Law, 5, 3-27. doi:10.1080/10683169908414991

Krackow, E., \& Lynn, S. (2003). Is There Touch in the Game of Twister®? The Effects of Innocuous Touch and Suggestive Questions on Children's Eyewitness Memory. Law and Human Behavior, 27, 589-604. doi:10.1023/B:LAHU.0000004888.52210.35

Krackow, E., \& Lynn, S. (2010). Event report training: A new intervention to improve children's eyewitness reports. Applied Cognitive Psychology, 24, 868-884. 
Krackow, E., Lynn, S., \& Payne, D. G. (2005). The death of Princess Diana: The effects of memory enhancement procedures on flashbulb memories. Imagination, Cognition and Personality, 25, 197-219. doi:10.2190/FBCF-T8VG-L5LJ-N8VL

Kully-Martens, K., Pei, J., Job, J., \& Rasmussen, C. (2012). Source monitoring in children with and without fetal alcohol spectrum disorders. Journal of Pediatric Psychology, 37, 725735. doi:10.1093/jpepsy/jsr123

Lane, S. M., Roussel, C. C., Villa, D., \& Morita, S. K. (2007). Features and feedback: Enhancing metamnemonic knowledge at retrieval reduces source-monitoring errors. Journal of Experimental Psychology: Learning, Memory, and Cognition, 33, 1131-1142. doi:10.1037/0278-7393.33.6.1131

Lindsay, D. S., \& Johnson, M. K. (1989). The eyewitness suggestibility effect and memory for source. Memory \& Cognition, 17, 349-358.

Lindsay, D. S., Johnson, M. K., \& Kwon, P. (1991). Developmental changes in memory source monitoring. Journal of Experimental Child Psychology, 52, 297-318. doi:10.1016/00220965(91)90065-Z

Loftus, E. F. (1979). Reactions to blatantly contradictory information. Memory \& Cognition, 7 , 368-374. doi:10.3758/BF03196941

Loftus, E. F., Miller, D. G., \& Burns, H. J. (1978). Semantic integration of verbal information into a visual memory. Journal of Experimental Psychology: Human Learning and Memory, 4, 19-31. doi:10.1037/0278-7393.4.1.19

Lorsbach, T. C., \& Ewing, R. H. (1995). Source monitoring in children with learning disabilities. International Journal of Disability, Development and Education, 42, 241-239. doi:10.1080/0156655950420306 
Lorsbach, T. C., Melendez, D. M., \& Carroll-Maher, A. (1991). Memory for source information in children with learning disabilities. Learning and Individual Differences, 3, 135-147. doi:10.1016/1041-6080(91)90013-Q

Martell, R. F., \& Evans, D. P. (2005). Source-Monitoring Training: Toward Reducing Rater Expectancy Effects in Behavioral Measurement. Journal of Applied Psychology, 90, 956963. doi:10.1037/0021-9010.90.5.956

Memon, A., Holley, A., Wark, L., Bull, R., \& Köhnken, G. (1996). Reducing suggestibility in child witness interviews. Applied Cognitive Psychology, 10, 503-518. doi:10.1002/(SICI)1099-0720(199612)10:6<503::AID-ACP416>3.0.CO;2-R

Memon, A., Meissner, C. A., \& Fraser, J. (2010). The Cognitive Interview: A meta-analytic review and study space analysis of the past 25 years. Psychology, Public Policy, and Law, 16, 340-372. doi:10.1037/a0020518

Milne, R., \& Bull, R. (2003). Does the cognitive interview help children to resist the effects of suggestive questioning? Legal and Criminological Psychology, 8, 21-38. doi:10.1348/135532503762871219

Poole, D., \& Lindsay, D. (2002). Reducing child witnesses' false reports of misinformation from parents. Journal of Experimental Child Psychology, 81, 117-140. doi:10.1006/jecp.2001.2648

Roberts, K. P., \& Powell, M. B. (2005). The relation between inhibitory control and children's eyewitness memory. Applied Cognitive Psychology, 19, 1003-1018. doi:10.1002/acp.1141 
Ruffman, T., Rustin, C., Garnham, W., \& Parkin, A. J. (2001). Source monitoring and false memories in children: Relation to certainty and executive functioning. Journal of Experimental Child Psychology, 80, 95-111. doi:10.1006/jecp.2001.2632

Rybash, J. M., \& Hrubi-Bopp, K. L. (2000). Source monitoring and false recollection: A life span developmental perspective. Experimental Aging Research, 26, 75-87. doi:10.1080/036107300243696

Saywitz, K. J., Geiselman, R., \& Bornstein, G. K. (1992). Effects of cognitive interviewing and practice on children's recall performance. Journal of Applied Psychology, 77, 744-756. doi:10.1037/0021-9010.77.5.744

Thierry, K. L. (2009). Practice retrieving source enhances young children's discrimination of live and story events. Journal of Applied Developmental Psychology, 30, 552-562. doi:10.1016/j.appdev.2008.12.030

Thierry, K. L., Lamb, M. E., Pipe, M., \& Spence, M. J. (2010). The flexibility of sourcemonitoring training: Reducing young children's source confusions. Applied Cognitive Psychology, 24, 626-644. doi:10.1002/acp.1574

Thierry, K. L., \& Spence, M. J. (2002). Source-monitoring training facilitates preschoolers' eyewitness memory performance. Developmental Psychology, 38, 428-437. doi:10.1037/0012-1649.38.3.428

Thierry, K. L., Spence, M. J., \& Memon, A. (2001). Before misinformation is encountered: Source monitoring decreases child witness suggestibility. Journal of Cognition and Development, 2, 1-26. doi:10.1207/S15327647JCD0201_1

Warren, A. R., Woodall, C. E., Thomas, M., Nunno, M., Keeney, J. M., Larson, S. M., \& Stadfeld, J. A. (1999). Assessing the effectiveness of a training program for interviewing 
child witnesses. Applied Developmental Science, 3, 128-135.

doi:10.1207/s1532480xads0302_6

Welch-Ross, M. K., Diecidue, K., \& Miller, S. A. (1997). Young children's understanding of conflicting mental representation predicts suggestibility. Developmental Psychology, 33, 43-53. doi:10.1037/0012-1649.33.1.43 
Table 1

Demographics of Study Sample

$N \quad \%$ of total sample

Gender

Male

$22 \quad 59.5$

Female

$15 \quad 40.5$

Age

4 years

17

45.9

5 years

20

54.1

Ethnicity

Black/African-American

Caucasian

29

78.4

Asian

1

2.7

Hispanic/Latino

2.7

Biracial

1

8.1

Other

3

5.4

Gross Family Income

$\begin{array}{lll}50,000-60,000 & 2 & 5.4 \\ 60,001-70,000 & 2 & 5.4 \\ 70,001-80,000 & 1 & 2.7 \\ 90,001-100,000 & 1 & 2.7 \\ \text { Over } 100,000 & 30 & 81.1\end{array}$

Primary Caregiver Highest Education Level

High School

$2 \quad 5.4$

Two-year College Degree

$1 \quad 2.7$

College

$11 \quad 29.7$

Master's

17

45.9

Doctorate

8.1

Professional School

3

8.1 
Table 2

Study Design by Condition

\begin{tabular}{|c|c|c|}
\hline Source Monitoring & Cognitive Interview & Control \\
\hline \multicolumn{3}{|c|}{ Session 1} \\
\hline \multicolumn{3}{|c|}{ Consent/Assent + Demographic Form + Viewing of Video 1 (to-be-remembered event) } \\
\hline \multicolumn{3}{|c|}{ Session 2} \\
\hline \multicolumn{3}{|c|}{ Source Monitoring Training or Control Procedure } \\
\hline $\begin{array}{l}\text { Source Monitoring } \\
\text { Training Procedure } \\
\text { - Viewing of Video } 2 \\
\text { (SM training video) } \\
\text { - Event Questions: } \\
\text { Brian played with some } \\
\text { blocks, didn't he? } \\
\text { - Source Questions: } \\
\text { Did I tell you Brian play } \\
\text { with some blocks or did } \\
\text { you see him play with the } \\
\text { blocks in the movie? }\end{array}$ & $\begin{array}{l}\text { Source Monitoring Control } \\
\text { Procedure } \\
\text { - Viewing of Video } 2 \\
\text { (SM training video) } \\
\text { - Event Questions: } \\
\text { Brian played with some } \\
\text { blocks, didn't he? }\end{array}$ & $\begin{array}{l}\text { Source Monitoring Control } \\
\text { Procedure } \\
\text { - Viewing of Video } 2 \\
\text { (SM training video) } \\
\text { - Event Questions: } \\
\text { Brian played with some } \\
\text { blocks, didn't he? }\end{array}$ \\
\hline \multicolumn{3}{|c|}{ Memory Interviews } \\
\hline $\begin{array}{l}\text { Standard Interview } \\
\text { (open-ended free recall } \\
\text { question) }\end{array}$ & Cognitive Interview & $\begin{array}{l}\text { Standard Interview } \\
\text { (open-ended free recall } \\
\text { question) }\end{array}$ \\
\hline \multicolumn{3}{|c|}{ "Yes/no" Leading Questions } \\
\hline \multicolumn{3}{|c|}{ Debriefing } \\
\hline
\end{tabular}

